\title{
PRIMARY LYMPHOPLASMACYTOMA OF THE CONJUNCTIVA
}

\author{
G. LUGASSY ${ }^{1}$, D. ROZENBAUM ${ }^{2}$, L. LIFSHITZ ${ }^{3}$, E. AVIEL ${ }^{2}$ \\ Ashkelon, Israel
}

\begin{abstract}
SUMMARY
We report the case of an 80-year-old patient with isolated lymphoplasmacytoma of the conjunctiva. Only five other such cases have been previously reported in the literature. In all six patients, the disease remained limited and no sign of systemic disease could be found after prolonged follow-up. As isolated plasmacytoma of the conjunctiva seems to be benign, treatment of such tumours should be conservative.
\end{abstract}

Lymphoplasmacytomas are rare monoclonal tumours consisting of lymphocytic infiltrates with plasmacytoid features and no evidence of systemic disease. The usual sites of such lesions are the respiratory tract, bones, lymph nodes and skin. ${ }^{1}$ They are rarely located in the eyes (2-5), and reports of isolated lymphoplasmacytomas of the conjunctiva are exceptional (6-8). We describe such a case and briefly review similar cases reported in the literature.

\section{CASE REPORT}

An 80-year-old man was referred to the Ophthalmology

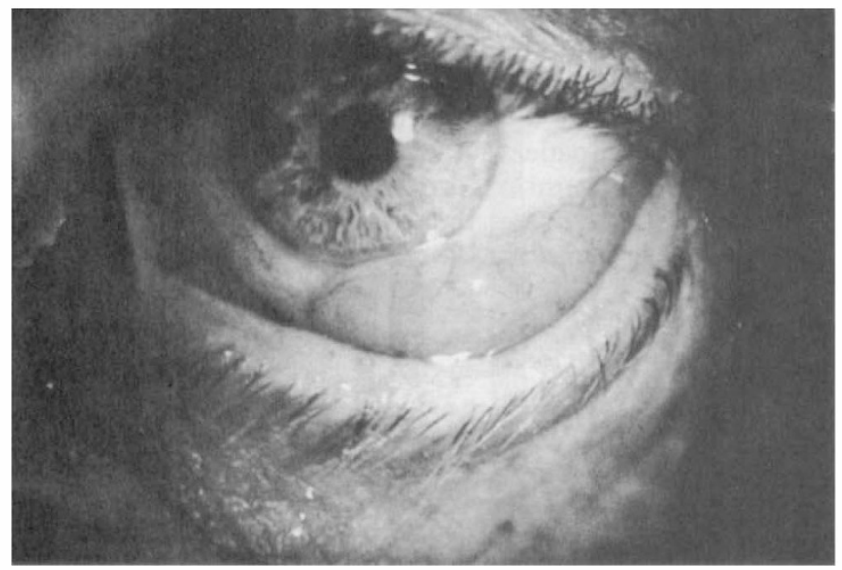

Fig. 1. Smooth tumour in bulbar conjunctiva.

From: 'Institute of Hematology, ${ }^{2}$ Department of Ophthalmology, and ${ }^{3}$ Institute of Pathology, Barzilai Medical Center, Ashkelon, Israel

Correspondence to: Dr G. Lugassy, Hematology Institute, Barzilai Medical Center, Ashkelon, Israel.
Department in August 1988 for resection of a left conjunctival tumour. His past history included no relevant findings.

Examination of the left eye revealed a solid, smooth, flesh-coloured conjunctival tumour in the lower fornix (Fig. 1). Complete ophthalmologic examination showed bilateral incipient cataract. The rest of the physical examination was normal. An excision of the tumour was performed under local anaesthesia, and the conjunctival defect was covered by a buccal mucosal graft. The postoperative course was uneventful.

On pathological examination, the histological picture was that of a lymphoplasmacytoid immunocytoma (Fig. 2) showing numerous PAS-positive intranuclear Dutcher bodies (Fig. 3a). Immunoperoxidase studies revealed a predominance of kappa-positive cells (Fig. 3b). Dutcher bodies also stained positively for kappa light chains. Compact sheets of cells showed positive staining for IgM, whereas only scattered cells were positive for IgG and IgA.

Since this histological picture is often associated with paraproteinemia, particularly of the Waldenstrom macro-

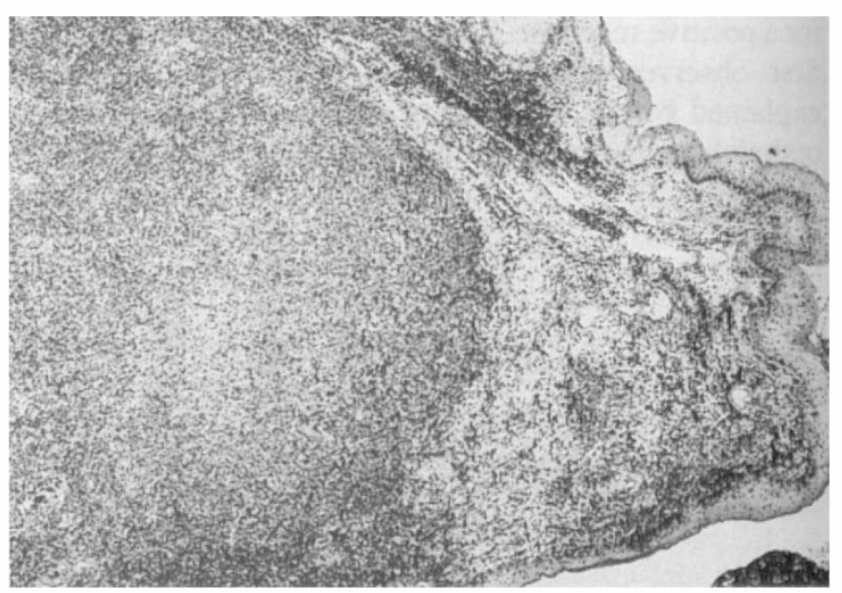

Fig. 2. Conjunctival tumour: infiltration by lymphoplasmacytoid cells $(\times 40)$. 


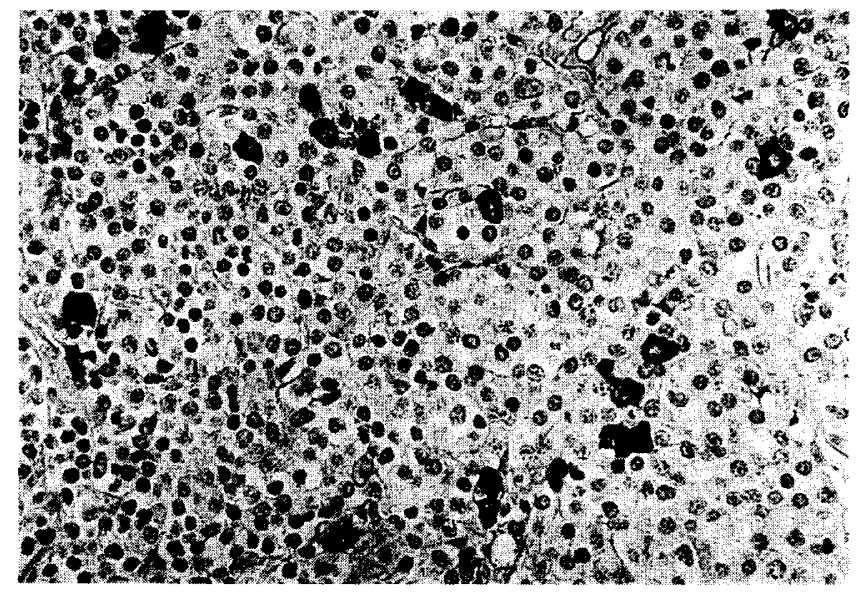

Fig. 3a. Infiltrate of lymphoplasmacytoid cells with PASpositive Dutcher bodies $(\times 300)$.

globulinaemia type, a complete staging was carried out. The erythrocyte sedimentation rate was 92 at the first hour. Peripheral blood count and liver and kidney function tests were all within normal limits. Electrophoresis of the serum portein showed no paraprotein, and no Bence Jones proteins were found in the urine. A chest X-ray and a CT scan of the orbit, the brain and the abdomen were all normal. A bone marrow biopsy showed a normal haematopoiesis with no plasma cell infiltration.

The patient was diagnosed as having an isolated lymphocytoma of the left conjunctiva. No specific therapy was given after excision. Repeated physical and ophthalmological examination and laboratory tests have failed to show any systemic involvement of the isolated tumour after 24 months of follow-up.

\section{DISCUSSION}

Isolated extramedullary plasmacytoma is a rare finding; three such cases are described for every 100 cases of multiple myeloma. Frequent sites of these tumours are the respiratory tract, lymph nodes, spleen and skin. ${ }^{1}$ Ocular plasmacytomas have been reported as an exceptional occurrence; the orbit is then the most frequent site. ${ }^{2-5}$

Our patient had an isolated plasmacytoma of the conjunctiva with no evidence of paraproteinaemia. The malignant nature of the tumour was confirmed by the monoclonality of the proliferative cells. To our knowledge, only five other cases of primary plasmacytomas of the conjunctiva have been previously reported in the literature. Jakobiec et al. ${ }^{6}$ described two such patients among 14 cases of ocular adnexal monoclonal lymphoid tumours; prolonged follow-up showed no evidence of systemic involvement in these two cases. Similarly, Vyas et al. ${ }^{7}$ reported a case of primary extramedullary solitary plasmacytoma of the conjunctiva with no extension of the disease during follow-up. Kremer et al, ${ }^{8}$ recently described two patients with different adnexal locations of localised extramedullary plasmacytomas, one under the conjunctiva of the caruncle, and the other under the tarsal conjunctiva. Evidence of multiple myeloma was not

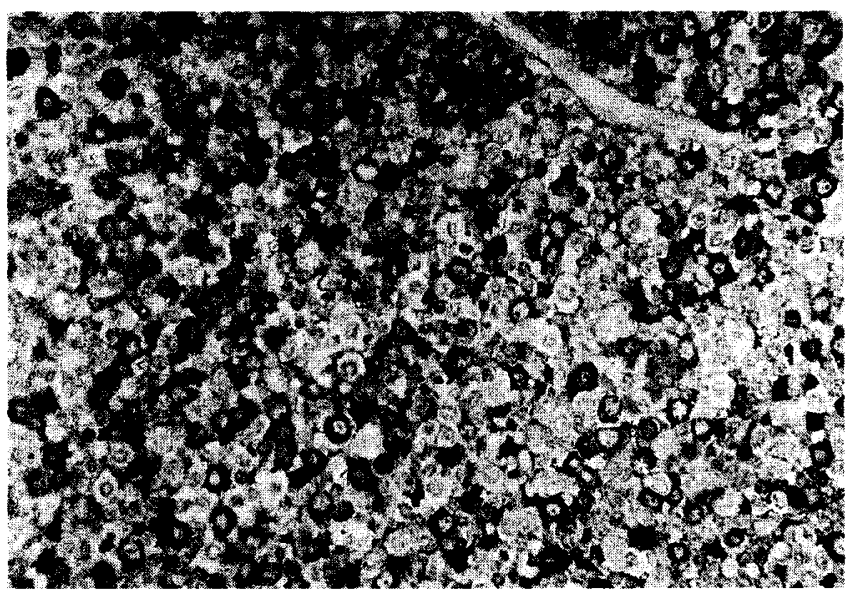

Fig. 3b. Immunoperoxidase staining showing a predominance of kappa positive cells $(\times 420)$.

found in either patient after a follow-up of six years. Medeiros et al. ${ }^{9}$ analysed the immunohistologic features of 61 patients with orbital and conjunctival lymphoid infiltrates. In only two patients could the process be characterised as a malignant lymphoid tumour of the conjunctiva. None had plasmacytoid features.

The natural history of primary extramedullary plasmacytoma is yet to be defined. Some remain limited for several years, while others progress to multiple myelomas. However, isolated plasmacytoma of the conjunctiva seems to have no propensity for developing into systemic disease and should therefore be treated conservatively.

Key words: Isolated plasmacytoma; Conjunctiva.

\section{REFERENCES}

1. Wiltshaw E: The natural history of extramedullary plasmacytoma and its relation to solitary myeloma of bone and myelomatosis. Medicine (Baltimore) 1976, 55: 217.

2. Jain BS: Solitary myeloma of the orbit. Am J Ophthalmol 1964, 58: 855-8.

3. Jim VKS: Plasmacytoma of the orbit. Am J Ophthalmol 1955, 39: 43-6.

4. Jampol LM, Marsh JC, Albert DM, Zimmerman LE. IgG associated lymphoplasmacytic tumour involving the conjunctiva, eyelid, and orbit. Am J Ophthalmol 1975, 79: 279-84.

5. Benjamin I, Taylor H, Spindler J. Orbital and conjunctival involvement in multiple myeloma. Report of a case. Am J Clin Pathol 1975, 63: 811-17.

6. Jakobiec FA, Iwamoto T, Patell M, Knowles II DM: Ocular adnexal monoclonal lymphoid tumours with a favourable prognosis. Ophthalmology 1986, 93: 1547-57.

7. Vyas MC, Vyas SP, Chandhary RK: Primary extramedullary solitary plasmacytoma. A case report. Indian J Ophthalmol 1984, 32: 181-3.

8. Kremer I, Flex D, Manor R. Solitary conjunctival extramedullary plasmacytoma. Ann Ophthalmol 1990, 22: $126-30$.

9. Medeiros LJ, Harmon DC, Linggood RM, Harris NC. Immunohistologic features predict clinical behaviour of orbital and conjunctival lymphoid infiltrates. Blood 1989. 74: $2121-9$. 